Projets

de paysage

\section{Projets de paysage}

Revue scientifique sur la conception et l'aménagement de l'espace

$5 \mid 2011$

Le jardin et ses rapports à l'art. Encadrer, décadrer, recadrer

\title{
Le jardin urbain de la Grande Bibliothèque : lieu ouvert et espace contesté
}

The urban park of the Grande Bibliothèque: Open place and contested space

Louis Jacob

\section{OpenEdition}

\section{Journals}

\section{Édition électronique}

URL : https://journals.openedition.org/paysage/21220

DOI : $10.4000 /$ paysage. 21220

ISSN : 1969-6124

Éditeur :

École nationale supérieure du paysage de Versailles-Marseille, Institut national des sciences appliquées Centre Val de Loire - École de la nature et du paysage, École nationale supérieure d'architecture et de paysage de Bordeaux, École nationale supérieure d'architecture et de paysage de Lille, Agrocampus Angers

\section{Référence électronique}

Louis Jacob, «Le jardin urbain de la Grande Bibliothèque : lieu ouvert et espace contesté », Projets de paysage [En ligne], 5 | 2011, mis en ligne le 16 janvier 2011, consulté le 16 octobre 2021. URL : http:// journals.openedition.org/paysage/21220 ; DOI : https://doi.org/10.4000/paysage.21220

Ce document a été généré automatiquement le 16 octobre 2021.

\section{(c) (i) (9)}

La revue Projets de paysage est mise à disposition selon les termes de la Licence Creative Commons Attribution - Pas d'Utilisation Commerciale - Pas de Modification 4.0 International. 


\title{
Le jardin urbain de la Grande Bibliothèque : lieu ouvert et espace contesté
}

The urban park of the Grande Bibliothèque: Open place and contested space

\author{
Louis Jacob
}

1 Je m'intéresse ici à ce qui me semble être un trait caractéristique du jardin urbain contemporain, à savoir sa capacité à nous offrir un coup d'œil sur le hors cadre et simultanément recadrer le paysage urbain. Je choisis délibérément de ne pas discuter directement des œuvres ou des pratiques artistiques qu'il intègre, avec lequel il entre en dialogue ou avec lequel il dispute l'espace urbain, mais plutôt de m'interroger sur ses dynamiques expérientielles, sociales et culturelles ${ }^{1}$.

2 Le concept de sédimentation est un des éléments clés permettant une analyse des qualités à la fois «médiales » et «spatiales » de la culture urbaine. Je l'entends en un sens qui nous provient à la fois de la phénoménologie et des sciences humaines (Bégout, 2005 ; Dilthey, 1988 ; Schütz, 1966). Il désigne le processus par lequel des expériences ou des communications sont objectivées et accumulées sous diverses formes de signes, d'images, d'états de conscience, d'objets, de dispositions et de comportements dans l'espace urbain; le concept recèle cependant des difficultés, comme l'avaient déjà entrevu Dilthey et Husserl, et l'on doit garder à l'esprit son caractère essentiellement dynamique (Bégout, 2005, p. 363-364).

3 Je soutiendrai que le jardin ou le parc urbain est révélateur des transformations actuelles de l'espace public. Plus largement, il engage un type d'expérience complexe et parfois ambivalent. Bien entendu, un long préambule pourrait s'avérer nécessaire afin d'identifier adéquatement les contraintes de l'aménagement et de la gouvernance territoriale des villes, mais je ne le ferai pas ici. Car si les sciences humaines nous ont habitués à découper et à déconstruire l'espace selon ses diverses dimensions matérielles, imaginaires, économiques, sociales ou politiques, je pense que le jardin urbain est aussi une excellente occasion de réfléchir à la phénoménologie quotidienne 
de l'espace, aux processus par lesquels ces diverses dimensions prennent corps et s'articulent dans l'expérience, en un lieu spécifique.

Il m'apparaît alors que les dimensions imaginaires, matérielles, voire politiques de l'espace urbain peuvent être décrites dans leur complémentarité, mais aussi de telle façon qu'on peut en faire ressortir les contradictions ou les ambivalences. C'est ce que je voudrais suggérer, en m'arrêtant principalement sur le cas du jardin urbain de la Grande Bibliothèque ${ }^{2}$. Il s'agit d'un espace somme toute modeste, dont la lecture ou l'interprétation, comme on le verra, peuvent sembler difficiles, mais un espace symptomatique, me semble-t-il, d'une situation plus générale.

\section{L'expérience du jardin et ses expressions littéraires}

5 Dans un premier temps, il convient de présenter et de contraster deux figures emblématiques, deux expressions littéraires du jardin. La première réside dans le fameux passage du roman épistolaire de Jean-Jacques Rousseau, Julie ou la Nouvelle Héloïse (1761), dans lequel Saint-Preux, qui se confie par lettre à son ami Édouard, raconte sa visite du jardin attenant au château de Clarens, un ancien verger complètement transformé. Le château est la demeure de Julie, son ancien amour, aujourd'hui mariée à un autre, par respect des conventions sociales et de la volonté de son père. Julie appelle pompeusement le jardin son «Élysée ». Saint-Preux accepte la promenade proposée par son rival, M. de Wolmar. Il écrit ceci : «Ce lieu, quoique tout proche de la maison, est tellement caché par l'allée couverte qui l'en sépare, qu'on ne l'aperçoit de nulle part. [...] En entrant dans ce prétendu verger, je fus frappé d'une agréable sensation de fraîcheur que d'obscurs ombrages, une verdure animée et vive, des fleurs éparses de tous côtés, un gazouillement d'eau courante, et le chant de mille oiseaux, portèrent à mon imagination du moins autant qu'à mes sens; mais en même temps je crus voir le lieu le plus sauvage, le plus solitaire de la nature, et il me semblait d'être le premier mortel qui jamais eût pénétré dans ce désert. » (Rousseau [1761], 1988, $4^{\text {e }}$ partie, lettre XI, p. 453-454.)

6 Accompagné de ses hôtes, Saint-Preux découvre progressivement le jardin. Puis il aura cette réflexion à l'adresse de $\mathrm{M}$. de Wolmar : « Il y a pourtant ici [...] une chose que je ne puis comprendre ; c'est qu'un lieu si différent de ce qu'il était ne peut être devenu ce qu'il est qu'avec de la culture et du soin : cependant je ne vois nulle part la moindre trace de culture; tout est verdoyant, frais, vigoureux, et la main du jardinier ne se montre point; rien ne dément l'idée d'une île déserte qui m'est venue en entrant, et je n'aperçois aucun pas d'hommes. - Ah! dit M. de Wolmar, c'est qu'on a pris grand soin de les effacer. » (Rousseau [1761], 1988, $4^{\mathrm{e}}$ partie, lettre XI, p. 461.)

7 Relatant la conversation et les explications de M. de Wolmar, la lettre de Saint-Preux, on le sait, prend les allures d'une philosophie romantique du jardin. L'enjeu en est assez clair: outre le déplacement sémantique qu'opère le $\mathrm{xvIII}^{\mathrm{e}}$ siècle sur la notion de " culture ", Rousseau en a contre le goût et les plaisirs factices du jardin classique, et rappelle les plaisirs vrais et simples de la nature, les vertus de la solitude et de la sincérité. Cela prend place dans un lieu qui semble refermé sur lui-même, comme le serait une île sauvage et déserte.

8 Par contraste, voici une autre figure du jardin, plus proche de nous, mais peut-être moins connue, qui se dessine dans un article du critique d'art Bernard Lamarche, publié 
dans le quotidien Le Devoir des 23 et 24 avril 2005. Le critique se prononce sur les œuvres intégrées à la Grande Bibliothèque, à la suite d'un concours lancé dans le cadre de la politique d'intégration des arts à l'architecture et à l'environnement du gouvernement du Québec. Outre les œuvres de Jean-Pierre Morin, de Dominique Blain et de Louise Viger, le critique commente les deux œuvres du sculpteur Roger Gaudreau qui prennent place dans le jardin extérieur, adjacent à la Bibliothèque :

Figure 1. Roger Gaudreau, Le Jardin civilisé, 2005 (béton, végétaux)

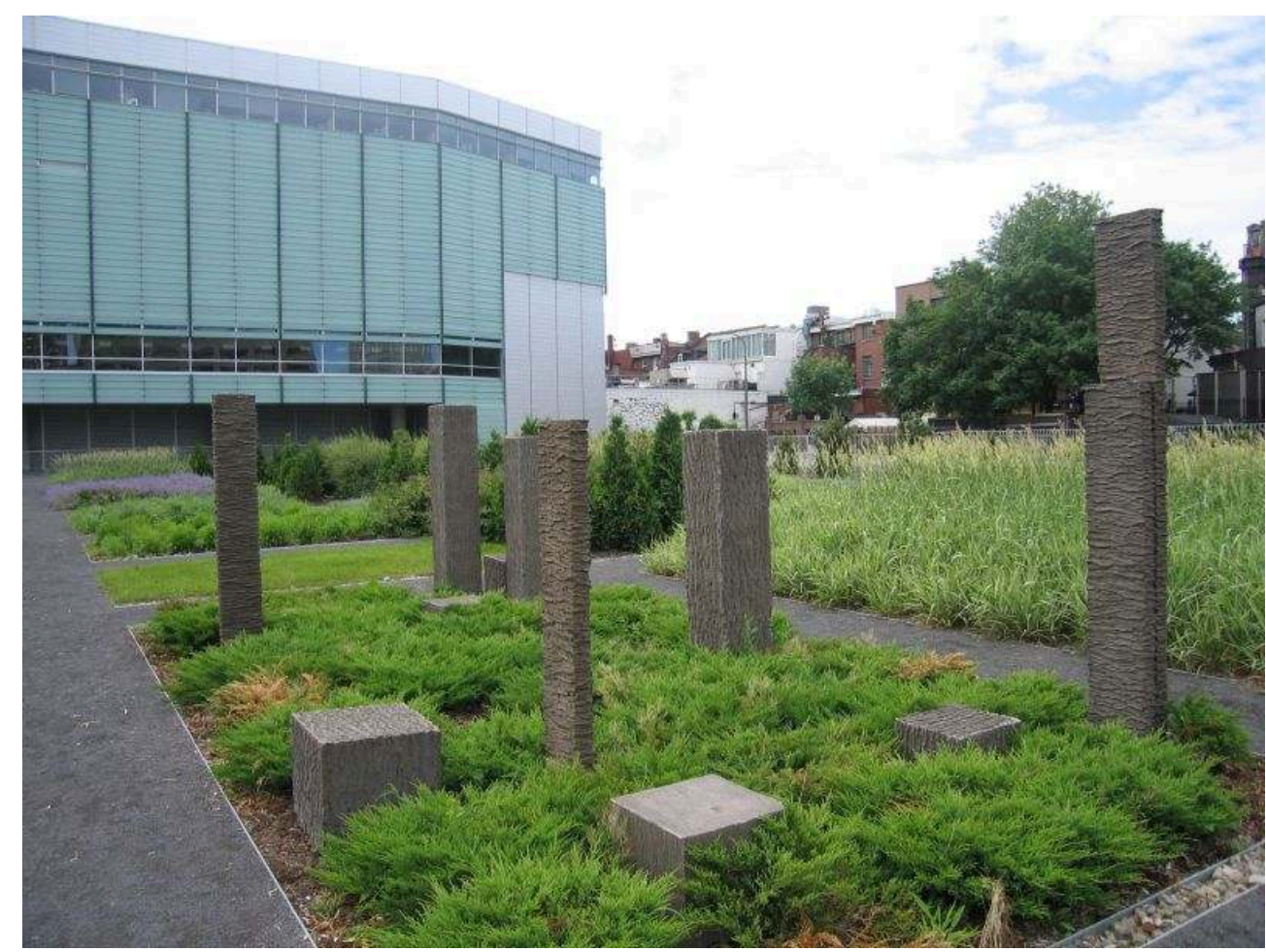

Roger Gaudreau. Grande Bibliothèque, Bibliothèque et Archives nationales du Québec, Montréal. Patkau/Croft Pelletier/Menkès Shooner Dagenais Architectes Associés.

Audrey Lalande, MCCCF. 
Figure 2. Roger Gaudreau, Le Jardin punk, 2005 (pierres, aluminium, végétaux)

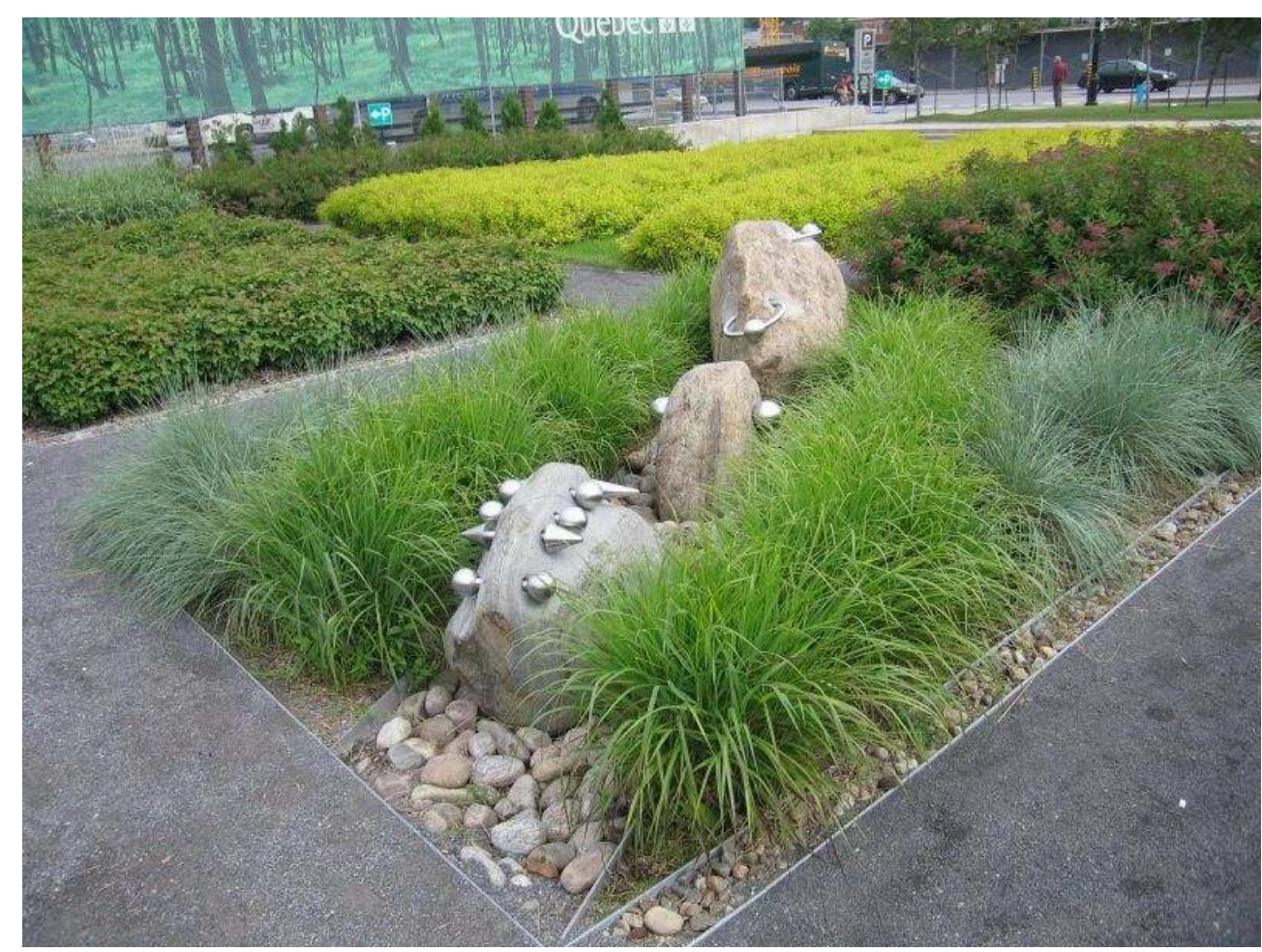

Roger Gaudreau. Grande Bibliothèque, Bibliothèque et Archives nationales du Québec, Montréal. Patkau/Croft Pelletier/Menkès Shooner Dagenais Architectes Associés.

Audrey Lalande, MCCCF.

«La contribution de Gaudreau est une demi-réussite. Son Jardin de la forêt urbaine, tout littéral soit-il, amuse à la vue de ces feuillages qui se retrouvent à la base d'arbres inversés dont les troncs rappellent la carrure des gratte-ciel. Ici, les façades (un sujet délicat autour de la BNQ $[s i c]^{3}$ ) miment des écorces d'arbre, comme si, à l'inverse de la BNQ [sic] justement, les tours se retrouvaient prises dans un écrin de bois.

«Cette allusion bellement réussie côtoie cependant un malheureux Jardin punk, à l'humour aussi flagrant que bête. Quelques pierres posées là ont été piercées. Gaudreau dit s'être inspiré de la jungle locale, telle qu'il l'a vue, en 2001, dans les images d'Yves Nantel diffusées par la revue Art Le Sabord. Plutôt que de se méfier du caractère hautement caricatural de ces images, l'artiste en a retenu un aspect, le piercing, qui n'est pourtant plus, depuis des lunes, l'apanage des seuls punks et qui en est encore moins la principale caractéristique. L'œuvre ne saurait être davantage inappropriée, dépassée et insensée. » (Lamarche, 2005, n.p.)

La question que je soulève ici en contrastant ces deux figures n'est pas de savoir, par exemple, si la panoplie du piercing que représente le sculpteur pour l'associer à la pierre et au jardin constitue réellement un répertoire d'objets en usage dans la jungle locale, ou si l'humour de l'artiste est plus judicieux quand vient le moment de railler les parements de verre de la Bibliothèque plutôt que d'entretenir un regard exotique sur la jeunesse qui erre sur les abords de la rue Ontario et sur la place Émilie-Gamelin (Straw, 2009). J'attire l'attention sur le fait que cette nouvelle expression de l'expérience du jardin révèle un espace discursif contradictoire et nous met en présence d'un « espace contesté » (Foucault, 2001 ; Harvey, 2006 ; Trom, 2001). 


\section{L'espace polyphonique du jardin urbain}

10 Il y d'abord le statut de l'œuvre d'art qui s'est considérablement déplacé. Depuis la fin du xxe siècle, la présence de l'art dans l'environnement urbain a été redynamisée avec les pratiques dites conceptuelles, la performance, l'installation, et toutes les déclinaisons de l'art qui se conçoit comme action, intervention, stratégie, manœuvre, ou relation... À Montréal seulement, on rappellera que la Biennale de 2004 portait sur le thème de l'espace public. Les événements Archi-Fiction, sous l'égide de la galerie Monopoli et de la Maison de l'architecture du Québec, proposent depuis plusieurs années des dialogues entre architectes, designers, écrivains et artistes visuels. Le centre d'information Artexte tenait également en 2004 un colloque sur l'art public qui comportait un volet artistique. Ajoutons encore les activités du centre de diffusion d'art multidisciplinaire Dare-Dare, qui combine des interventions artistiques, des colloques, des pratiques participatives ou des activités de médiation culturelle, mettant à contribution plusieurs artistes, des professionnels, des citoyens et des publics très variés, préoccupés par la vie civique et nos façons d'habiter les espaces urbains. La galerie Vox, pendant l'été 2008, proposait une exposition, une table ronde et des ateliers de discussion où était mis en question le processus de revitalisation du Quartier des spectacles.

11 D'autres initiatives, comme celles de Paysages Éphémères sur l'avenue du Mont-Royal, ou celles d'Artefact sur le canal de Lachine, dans le parc du Mont-Royal et sur l'île Sainte-Hélène, comme les sites du Musée d'art urbain ou les interventions photographiques du Mouvement art public, sont aussi à compter dans ce dynamisme très particulier d'une réflexion élargie sur l'environnement urbain. Cette série d'événements n'est évidemment pas close, et elle continue.

Ces pratiques artistiques contemporaines nous invitent bien sûr à explorer sans cesse de nouvelles formes de participation à la vie culturelle, mais elles incitent aussi à un scepticisme salutaire envers les idées trop rapidement servies autour du développement, du renouveau urbain, ou encore de ce qu'on pourra appeler la ville spectaculaire.

13 Dans le jardin de la Grande Bibliothèque, l'œuvre est censée agir, elle est censée apporter un point de vue distancié et critique par rapport à son propre lieu d'inscription; les explorations artistiques $d u x^{e}$ siècle et l'évolution récente de l'art public préparent le terrain en quelque sorte pour une œuvre autonome, réflexive, à laquelle n'est assignée aucune fonction sociale parfaitement évidente. Dans cet espace discursif, le critique Bernard Lamarche a donc parfaitement le droit de se montrer déçu. Nous y reviendrons. Mais le texte nous met en présence de discours hétéronomes qui ne sont pas la chasse gardée de l'esthétique et du jugement de goût. Quel est le peuple qui habite cette « jungle locale»? Qui produit les images légitimes des habitants du quartier? Que racontent les résidents, les itinérants, les travailleurs, les voyageurs, les usagers de notre jardin urbain?

14 Je crois que les deux expressions littéraires du jardin brièvement évoquées ici sont révélatrices de médiations symboliques sur lesquelles nous devons maintenant nous arrêter. Il s'agit bien entendu de deux expressions qui prennent place dans des espaces discursifs fort différents, et deux époques, deux genres, deux mondes si on veut, mais ces deux figures ne sont pas incommensurables, comme je voudrais le démontrer. Dans les deux expressions, nous avons en effet affaire à une expérience pleine de sens, 
l'expérience d'un espace singulier construit ou structuré par un sujet qui fait l'épreuve d'un lieu et qui conçoit des attentes particulières à l'égard de ce lieu. Entre le jardin que découvre Saint-Preux et celui de la rue Berri, l'expérience du jardin a changé de forme et de contenu, mais il s'agit toujours d'une expérience subjective, nourrie de multiples médiations symboliques, propres cette fois à l'agglomération urbaine du Xxi ${ }^{e}$ siècle.

\section{Un lieu ouvert, un espace contesté}

Le jardin urbain de la Grande Bibliothèque est un jardin évolutif, aménagé sur l'esplanade du côté nord de l'édifice, à l'angle des rues Berri et Ontario. Il est réalisé sous la direction du même consortium à qui l'on doit la Bibliothèque : Patkau/Croft Pelletier/Menkès Shooner Dagenais. Il est divisé en vingt-neuf lots ou modules - pour faire écho aux vingt-neuf sections de l'édifice - disposés selon une matrice variable, parcourue de passages rectilignes relativement étroits. Cette division en lots évoquerait aussi, selon les concepteurs, les jardins communautaires de Montréal (BAnQ, 2004 et 2005). La végétation est dense, et comporte des arbustes, des graminées, et des arrangements de conifères, bien visibles notamment le long de l'avenue Savoie. Le jardin est dit évolutif puisque à l'origine, on annonçait vouloir en faire un espace dans lequel prendrait place une collection d'œuvres acquises par voie de concours au fil des ans. On envisageait également la tenue d'activités d'animation (BAnQ, 2004).

Le jardin est accessible de l'intérieur de la Bibliothèque en empruntant la porte nord. Il s'offre également à la vue des usagers de la Bibliothèque, notamment à travers la paroi vitrée du niveau 1. Les accès au niveau de la rue sont multiples; on peut aborder et pénétrer le jardin de toutes parts, par exemple en empruntant la volée de marches qui le borde sur toute sa longueur du coté est. Sur l'esplanade, entre la porte nord et le jardin proprement dit, on trouve une aire de stationnement pour vélos. Une chaussée borde le jardin sur son côté nord, et traverse perpendiculairement l'esplanade pour descendre ensuite le long de l'avenue Savoie du côté ouest, vers le stationnement souterrain. Au-delà de cette chaussée a été aménagé un terrain gazonné qui couvre tout le reste du quadrilatère. Le terrain est agrémenté de légers monticules sphériques, et il est à son tour traversé par deux chemins en diagonale reliant la rue Ontario et se croisant en son centre. 
Figure 3.

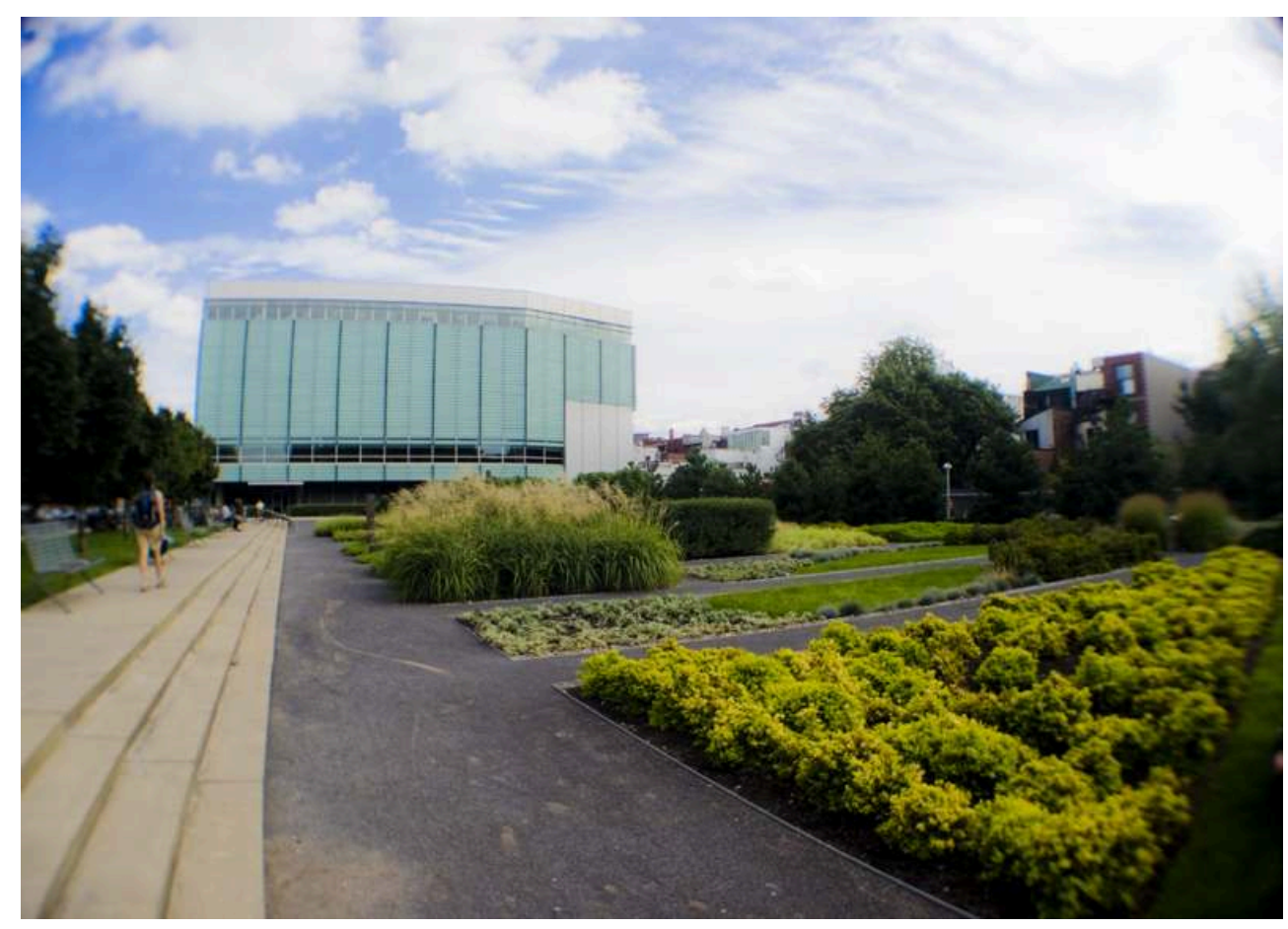

Martin Rose, 2010.

17 Je présume qu'il se trouvera bien peu d'usagers ou de visiteurs qui se surprendraient comme Saint-Preux de tomber dans un «coin de nature, non cultivé » comme «en dehors du monde ". Au contraire, nous nous attendons plutôt à trouver un lieu ouvert sur la ville, intégré au tissu urbain, multifonctionnel, où coexistent différents usages. Ces attentes sont propres à plusieurs espaces publics. J'ai déjà eu l'occasion de m'exprimer sur ce que j'entends par espace public (Jacob, 2008) : l'espace public ne doit pas être confondu avec la place publique elle-même, qui n'est que l'une de ses expressions. Contrairement à l'idée répandue, l'espace public n'est pas nécessairement un lieu consensuel, mais au contraire, un lieu où se font voir et entendre les divergences, les désaccords. C'est le lieu de la pluralité (Germain, 2000 ; Ghorra-Gobin, 2001). Ces lieux que nous persistons à appeler espaces publics le sont, souvent, de moins en moins; ce sont des lieux soumis à des pressions très fortes, réduits à des fonctions utilitaires contraires à l'idée de représentation, de mouvement ou de dialogue citoyen (Berdoulay et al., 2004).

Aujourd'hui, notre réflexion sur l'espace public inclut forcément, outre les dimensions classiques qui ont trait à l'organisation des flux, à l'environnement bâti et à la représentation du pouvoir légitime, ces autres dimensions fondamentales, révélées de manière exemplaire dans les pratiques artistiques contemporaines: les usages populaires ou quotidiens, les relations sociales, les savoirs, les normes et les symboles de la vie urbaine, bref, la mise en scène de la socialité et de la civilité, avec ses contradictions et ses conflits.

19 La ville s'offre à nous comme un ensemble de lieux potentiels, d'espaces " feuilletés » ou « multicouches » à travers lesquels nous devrions apprendre à nous déplacer et qu'il faudrait nous réapproprier (Nicolas-Le Strat, 2006). C'est la raison ultime des nouveaux espaces publics qui misent sur la perméabilité, la connexion, la polyphonie, pour 
reprendre quelques-uns des termes clés de ce paradigme architectural contemporain. L'une des vertus du jardin de la Grande Bibliothèque est contextuelle : il nous donne à voir, sur un mode ironique, la structure inachevée de l'îlot Voyageur ${ }^{4}$, de l'autre côté de la rue, et il force les liens entre les populations disparates du quartier.

Figure 4.

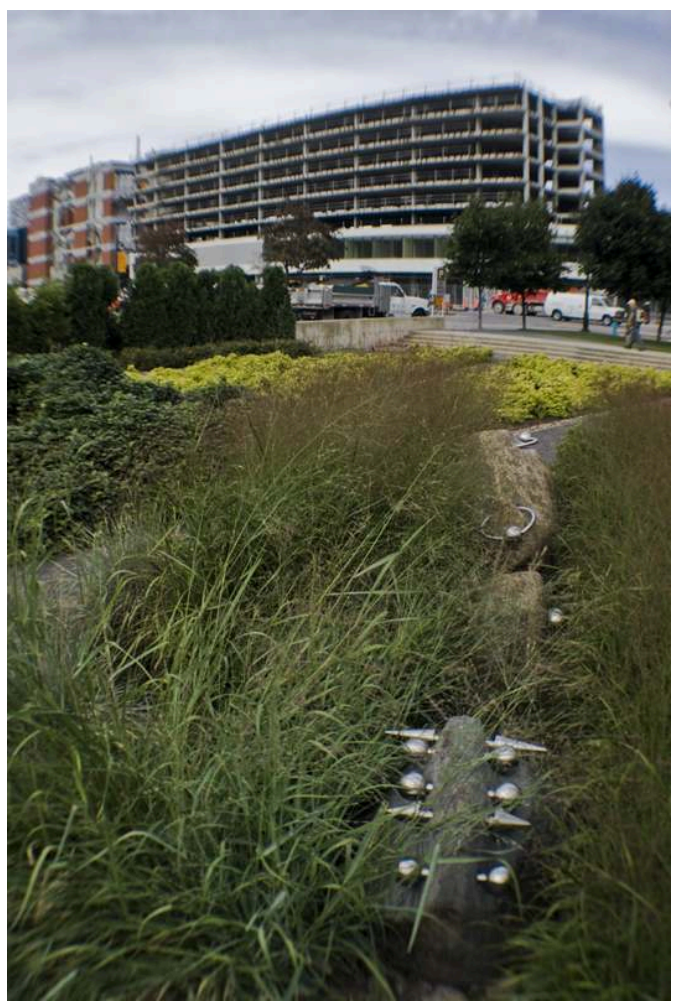

Martin Rose, 2010

\section{Une déambulation, et un essai d'interprétation}

Je mets maintenant entre parenthèses ces questions par ailleurs cruciales qui concernent le jardin en tant qu'espace public. On devrait en effet s'interroger sur le cadre juridique et réglementaire qui balise la liberté de mouvement et protège ce qu'il est convenu d'appeler la paix publique. On pourrait aussi questionner les modes d'appropriation du lieu, les usages, la circulation piétonnière, les microsocialités, les récits et les parcours habituels du lieu. Je laisse également de côté l'histoire spécifique du lieu, le processus qui mène à la localisation de la Bibliothèque en ce lieu précis, de même que le programme particulier du consortium Patkau/Croft Pelletier/Menkès Schooner Dagenais ou celui du sculpteur Roger Gaudreau.

21 Revenons plutôt à l'expérience du jardin qui ne s'est exprimée que très partiellement encore dans la critique de Bernard Lamarche. Un autre critique pourrait regretter par exemple que l'intervention artistique n'ait pas pris la voie de l'art relationnel ou de l'art participatif, un autre aurait souhaité un projet culturel dans lequel se seraient directement impliqués les gens du coin (Borasi et Zardini, 2008; Pelletier, 2008; Pietromarchi, 2005). L'œuvre de Gaudreau et la polémique à peine soulevée par le critique ont rapidement été ensevelies sous d'autres sédimentations; la végétation 
croît en densité et en hauteur autour des sculptures, les usagers s'approprient l'espace. Essentiellement, le propre du jardin urbain tel que je le conçois est de se tourner vers le dehors, ou de relancer la dialectique du dedans et du dehors, et ainsi recadrer l'ensemble de la ville.

Figure 5.

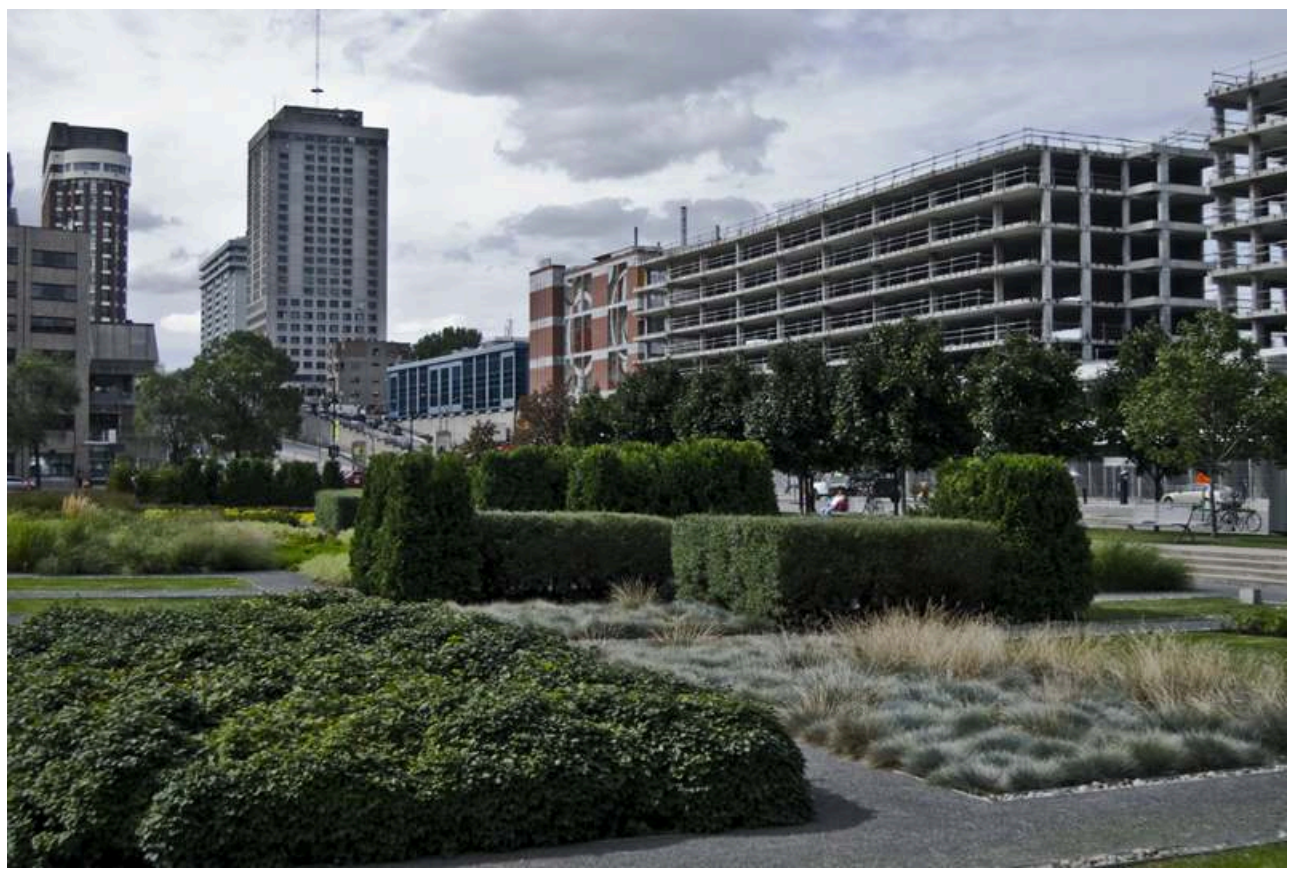

Martin Rose, 2010

Le texte de Lamarche et son espace discursif polyphonique nous renvoient donc invariablement à l'expérience du marcheur dans la ville qui traverse des espaces contrastés et enchaînés (Sansot, 1986). Par exemple, lorsque je descends la côte de la rue Berri à partir de la rue Sherbrooke, je domine la grande pelouse barrée d'un X, et je pourrais presque deviner ce que contiennent les modules végétaux du jardin. Le jardin est quasiment lisible, même de loin, alors que si je l'aborde en provenant de l'est, sur la rue Ontario, je serais surtout gêné par les chantiers de construction. Le jardin est même un peu dissimulé en raison de la topographie qui m'empêche de voir ce qui se passe un peu plus loin, au-delà de la chaussée, du trottoir opposé sur la rue Berri, et de la volée d'escaliers. Les habitués qui passent par la petite avenue Savoie ou qui proviennent de la rue Saint-Denis en empruntant la rue Ontario apprécieront peut-être mieux les monticules lunaires de la pelouse. 
Figure 6.

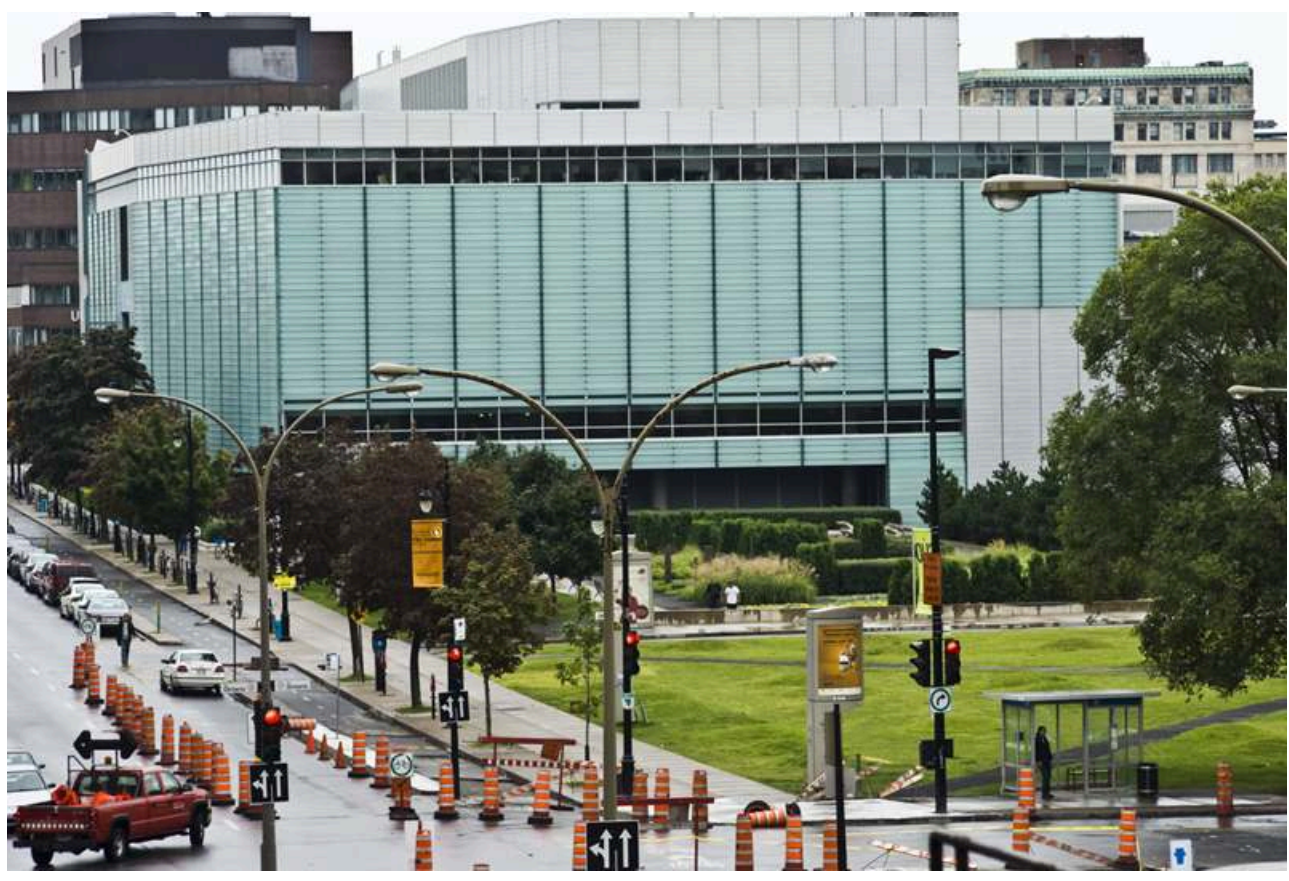

Martin Rose, 2010.

Si je poursuis ce genre d'exploration en arpentant la rue Berri mais du côté est, en direction de la place Émilie-Gamelin, et que je m'arrête pour contempler la Grande Bibliothèque et son jardin, alors je peux vraiment entrevoir le caractère rassurant $\mathrm{du}$ jardin. Ce caractère rassurant réside dans ce qui va apparaitre comme une allégorie ; le jardin constitue en effet une allégorie de la Bibliothèque, en partie parce qu'il a été voulu ainsi, par sa division en vingt-neuf lots, par le choix des matériaux et des plantations, par le choix de l'œuvre d'art à laquelle on demandait d'évoquer les collections qui se trouvent à l'intérieur du bâtiment (Gouvernement du Québec, 2008; BAnQ, 2004). Vu de l'autre côté de la rue Berri, en tournant le dos au désastre de l'îlot Voyageur, on comprendra peut-être qu'on peut y circuler comme entre les rayons d'une bibliothèque. Même si le visiteur pense bien qu'il n'y a rien à retirer des rayons comme un livre qu'il pourrait ouvrir, n'importe lequel, et emporter avec soi -, rien vraiment sur quoi se pencher sinon les plantations denses entre les allées étroites, l'expérience qu'offre le jardin reste une sorte d'invitation, une invitation qu'il peut accepter.

24 Un espace clair et manifeste, avec des régularités autres que celles de la rue, des végétaux presque individués, distribués de façon savante dans des modules touffus c'est tout de même une surprise dans la trame urbaine du centre-ville qui cherche à s'étendre depuis des années vers l'est. La circulation à l'intérieur du jardin, en bas des quelques marches, n'est sans doute pas très libre, mais cette circulation miniature et apaisante tranche avec le joyeux chaos de la ville. C'est un autre aspect de «la vie de l'esprit » si on veut, qui procède par série, par paquet, qui crée des ensembles. Quelque chose d'inusité dans la ville, justement parce que ordonné sans être parfaitement linéaire, à l'image des rayons d'une bibliothèque. La curiosité pousse à chercher la clé, non pas la raison, mais le quoi et le comment qui permettront au visiteur de s'adonner à cet espace indéterminé. 
Figure 7.

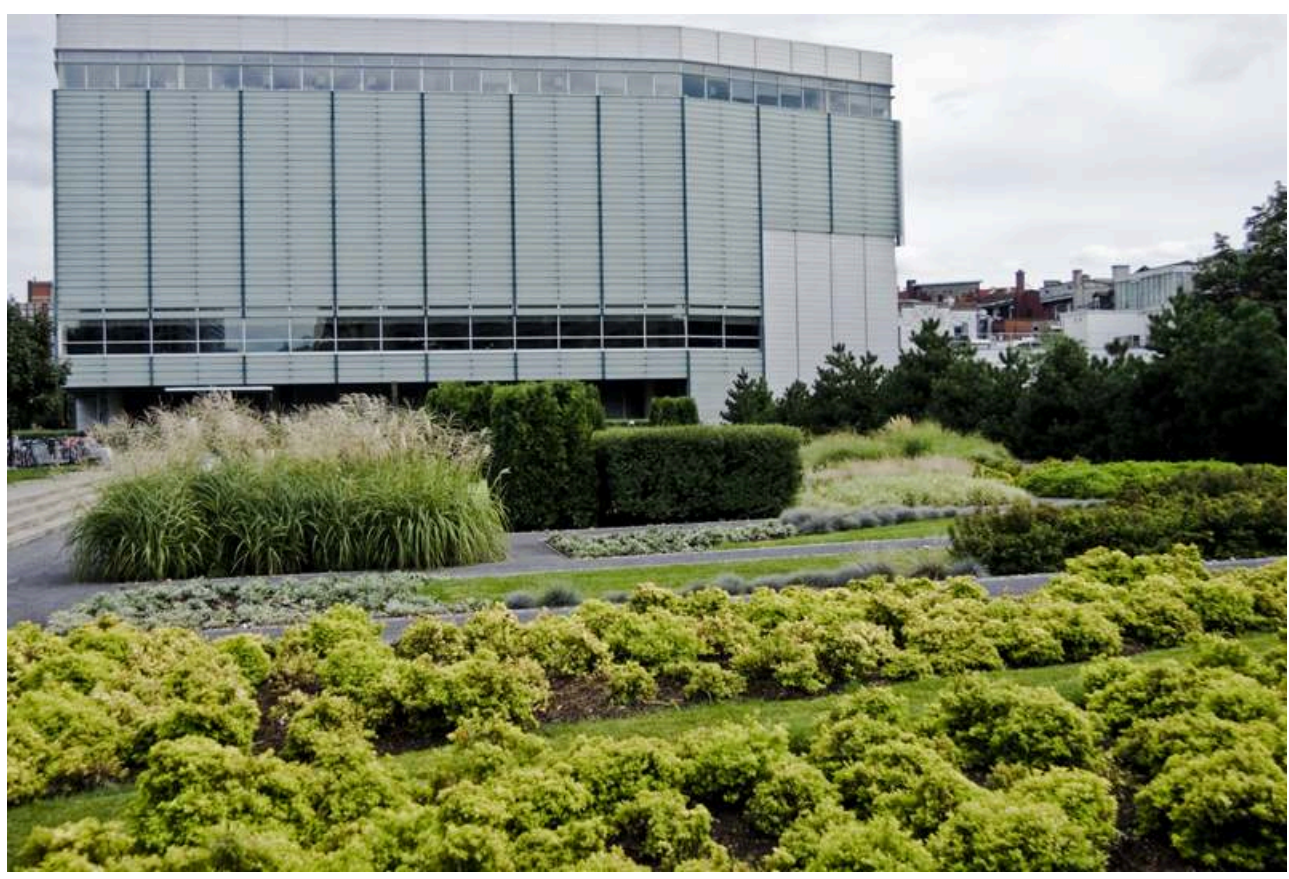

Martin Rose, 2010.

Dans un tel jardin, on pourra rechercher, non pas la solitude et la jouissance des plaisirs authentiques de la conversation entre amis ou avec les enfants, comme le préconisait Rousseau, mais rechercher un moment de repos sur l'heure du lunch, une pause dans la déambulation urbaine, un lieu de transit au sortir de l'autobus qui nous débarque à Montréal, mille façons de s'approprier la ville et, pourquoi pas, de se perdre dans l'anonymat de la foule. Le jardin de la Grande Bibliothèque est bien un jardin, mais c'est aussi un espace public urbain qui obéit à une autre logique et qui génère d'autres expériences que celles du petit Élysée de Julie et de M. de Wolmar. Ce jardin est entre autre chose une allégorie, retravaillée par l'intervention artistique et par les sédimentations de sens multiples que provoque la ville. Pour paraphraser Pierre Sansot, ce jardin n'est pas en dehors de « la mouvance de la ville». C'est un objet urbain instable (Sansot, 2004). 
Figure 8.

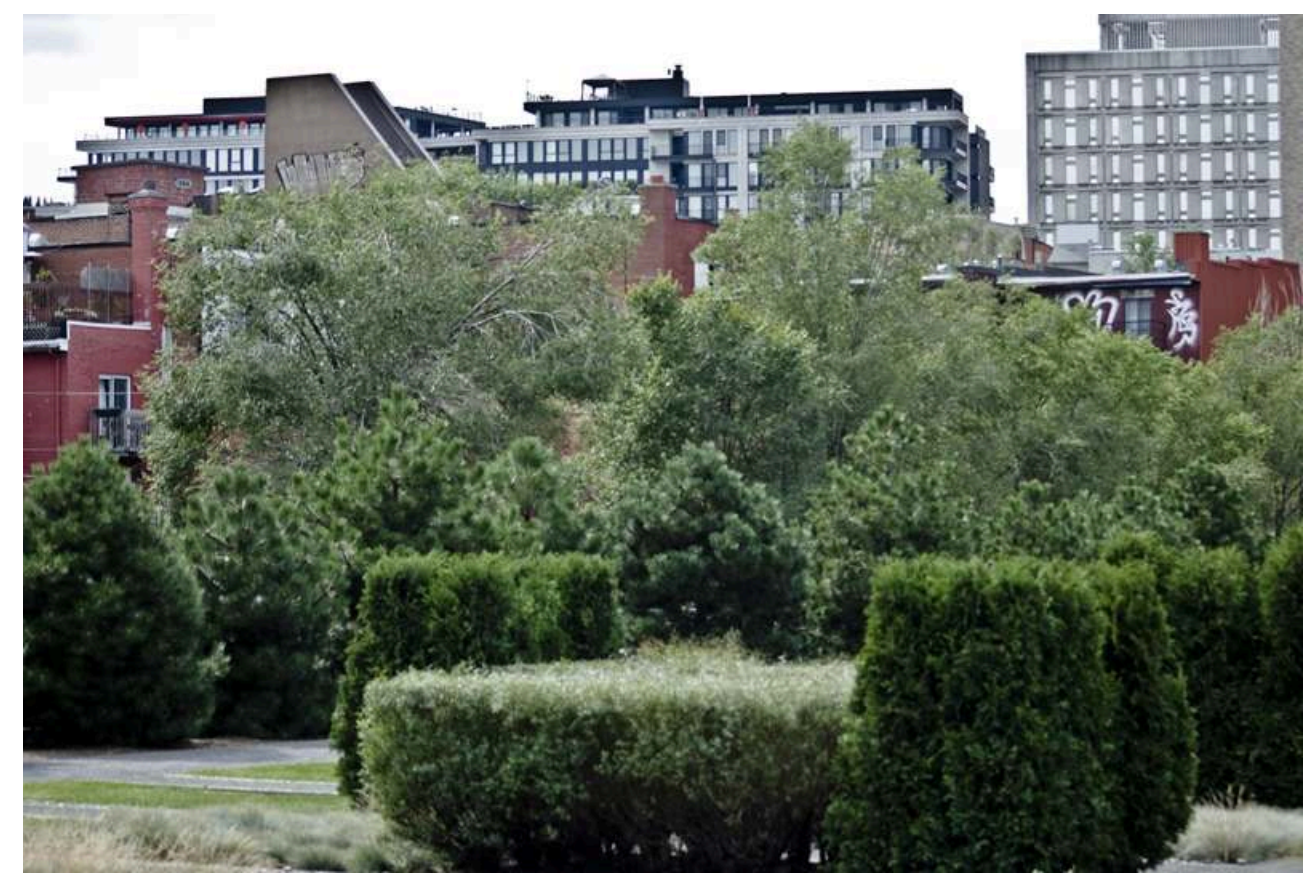

Martin Rose, 2010. l'espace public communicationnel, la ville médiale ou polyphonique, etc.) sont désormais disponibles, elles existent, bien qu'elles ne soient pas toutes également sollicitées par les usagers du jardin. Elles sont sédimentées, et leur réactualisation dépend d'une foule de facteurs exogènes, dont celui d'un mode de production de l'espace qui échappe au seul point de vue de l'acteur individuel (Bégout, 2005).

Pour conclure, je pense qu'on ne se trompe pas en considérant que le jardin urbain de la Grande Bibliothèque, quel que soit notre jugement quant à sa réussite et à ses développements futurs, est à bien des égards un puissant analyseur, une sorte de laboratoire : les stratégies artistiques, les contraintes de la trame urbaine, les projets de développement et de revitalisation des îlots adjacents, les usages et les mémoires du lieu, toutes ces dimensions forcent à un "recadrage " non seulement du jardin luimême en tant que configuration significative, mais du spectacle de la ville qui se déroule dedans et dehors. En outre, il est bien possible, comme le faisait remarquer encore tout récemment Luc Lévesque, que la nature du territoire et des relations urbaines demande des interventions plus souples et moins contraignantes, mais complémentaires aux grands programmes d'aménagement ou de revitalisation (Lévesque, 2008, p. 162). 


\section{BIBLIOGRAPHIE}

Bégout, B., La Découverte du quotidien, Paris, Allia, 2005.

Berdoulay, V., Da Costa Gomes, P . C., et Lolive, J. (sous la dir. de), L'Espace public à l'épreuve : régressions et émergences, Pessac, Maison des sciences de l'homme d'Aquitaine, 2004.

Bibliothèque et Archives nationales du Québec (BAnQ), À rayons ouverts, chroniques de la Bibliothèque nationale du Québec, Montréal, direction des Communications et des Relations publiques de la Bibliothèque nationale du Québec, $n^{\circ}$ 61, automne 2004.

Bibliothèque et Archives nationales du Québec (BAnQ), Voici votre Grande Bibliothèque, Montréal, direction des Communications et des Relations publiques de la Bibliothèque nationale du Québec, 2005.

Borasi, G. et Zardini, M. (sous la dir. de), Actions : comment s'approprier la ville, Montréal, Centre canadien d'architecture, 2008.

Boutros, A. et Straw, W. «Introduction », dans Circulation and the City : Essays on Urban Culture, Montréal, McGill-Queen's University Press, 2010, p. 3-20.

Dilthey, W., L'Édification du monde historique dans les sciences de l'esprit, traduit de l'allemand (1910), présentation et notes par S. Mesure, Paris, Les Éditions du Cerf, 1988.

Doevendans, K. et Schram, A., « Creation/Accumulation City », Theory, Culture \& Society, $\mathrm{n}^{\circ} 2,2005$, vol. 22, p. 29-43.

Foucault, M., « Espace, savoir et pouvoir », et « Des espaces autres », dans Dits et écrits, Paris, Gallimard, 2001, t. II, p. 1089-1104, 1571-1581.

Fuller, M., « Art Methodologies in Media Ecology », dans O'Sullivan, S. et Zepke, S. (ed.), Deleuze, Guattari, and the Production of the New, Londres, Continuum, 2008.

Gaonkar, D. P. et Povinelli, E. A., « Technologies of Public Forms : Circulation, Transfiguration, Recognition », Public Culture, n 3, 2003, vol. 15, p. 385-397.,

Germain, A., « Des parcs au pluriel : penser la diversité dans l'aménagement des espaces publics ", colloque « La diversité culturelle à travers la nature », Jardin botanique de Montréal, 2000.

Ghorra-Gobin, C. (sous la dir. de), Réinventer le sens de la ville : les espaces publics à l'heure globale, Paris, L'Harmattan, 2001.

Gouvernement du Québec, Bilan 2004-2007. Intégration des arts à l'architecture et à l'environnement, Québec, ministère de la Culture, des Communications et de la Condition féminine du Québec, 2008.

Harvey, D., « Space as a Key Word », dans Spaces of Global Capitalism : Towards a Theory of Uneven Geographical Development, Londres et New York, Verso, 2006, p. 119-148.

Jacob, L., « Espaces publics, lieux de la pluralité », dans Caissie, J.-P. et al., Dis/location 1, projet d'articulation urbaine : Square Viger, Montréal, Dare-dare, 2008, p. 103-111.

Lamarche B., « Euvres de passage », Le Devoir, 23-24 avril 2005. Consulté en ligne le 15 septembre 2010, URL : http://www.ledevoir.com/culture/arts-visuels/79981/oeuvres-de-passage

Lévesque, L., « La place publique comme constellation interstitielle : parcours historique et expérimentations ", dans Jébrak, J. et Julien, B. (sous la dir. de), Les Temps de l'espace public : 
construction, transformation et utilisation, Québec, Éditions Multimondes, coll. « Cahiers de l'Institut du patrimoine de l'UQÀM », 2008, p. 145-169.

Mons, A., «L'intervalle des lieux », Le Portique, $\mathrm{n}^{\circ}$ 12, 2003. Consulté en ligne le 15 septembre 2010, URL : http://leportique.revues.org/index578.html

Nicolas-Le Strat, P., « Multiplicité interstitielle », 2006. Consulté en ligne le 15 septembre 2010 sur le site de l'Institut social et coopératif de recherche appliquée (Iscra), URL : https://

www.iscra.org/

Pelletier, S. (Sous la dir. de), ATSA : Quand l'art passe à l'action, Montréal, Action terroriste socialement acceptable, 2008.

Pietromarchi, B. (ed.), The [Un]Common Place: Art, Public Space and Urban Æsthetics in Europe, Barcelone, Actar, 2005.

Quintas, E. (sous la dir. de), Culture pour tous, Montréal, Le Sabord, 2007.

Rousseau, J.-J., Julie ou la Nouvelle Héloïse. Lettres de deux amants habitants d'une petite ville au pied des Alpes, (1761), intro., chrono., biblio., notes et choix de variantes par R. Pomeau, édition augmentée, revue et mise à jour, Paris, Bordas, 1988.

Sansot, P., « Une étude attentionnée d'un fragment urbain », Les Formes sensibles de la vie sociale, Paris, PUF, 1986, p. 165-181.

Sansot, P., Poétique de la ville (1996), Paris, Payot, 2004.

Schütz, A., Collected Papers I : The Problem of Social Reality, articles rassemblés par M.A. Natanson et H.L. van Breda, Dordrecht, Martinus Nijhoff, 1966.

Trom, D., « Comment décrire un objet disputé ? ", dans Benoist, J. et Karsenti, B. (sous la dir. de), Phénoménologie et Sociologie, Paris, PUF, 2001, p. 65-81.

Straw, W., "The Palace, the Terminal and the Park: Three Blocks in the Middle of Montreal », dans Straw, W. et Tallack, D. (ed.), Global Cities/Local Sites, Melbourne, Melbourne University Publishing, 2009.

\section{NOTES}

1. Ma réflexion puise à diverses sources, dont les travaux du groupe de recherche Médialité et vie urbaine, qui portent sur les processus de sédimentation, de circulation et de production de la culture urbaine. On trouvera un bref aperçu de la problématique générale dans Boutros et Straw, 2010. Voir également Doevendans et Schram, 2005 ; Fuller, 2008 ; Gaonkar et Povinelli, 2003.

2. La Grande Bibliothèque, située dans le centre-ville de Montréal, est l'édifice principal de la BAnQ (Bibliothèque et Archives nationales du Québec).

3. Bibliothèque nationale du Québec. Le sigle exact est $B A n Q$, pour Bibliothèques et Archives nationales du Québec.

4. Dans un contexte de sous-financement public, et pour remédier au manque de locaux, l'université du Québec à Montréal s'était engagée en 2005 dans un projet immobilier en partenariat avec la firme Busac. Le projet qui comportait à l'origine, en plus du pavillon et des résidences universitaires, une tour à bureaux, une gare d'autobus ainsi que des stationnements souterrains, s'est avéré une catastrophe, tant financière que politique. Certains travaux seront suspendus, seuls la gare et les stationnements seront complétés. 


\section{RÉSUMÉS}

La problématique générale de la médialité questionne les processus de sédimentation, de circulation et de production de la culture urbaine. À partir du jardin urbain de la Grande Bibliothèque, dans le centre-ville de Montréal, ce sont les transformations actuelles de l'espace public, et sa phénoménologie quotidienne, qui sont aussi interrogées. Ce jardin urbain est un lieu ouvert sur la ville et il prend place dans l'espace discursif contradictoire. Les stratégies artistiques, les contraintes de la trame urbaine, les projets de développement et de revitalisation des îlots adjacents, les usages et les mémoires du lieu, toutes ces dimensions forcent à un « recadrage » non seulement du jardin lui-même en tant que configuration significative, mais du spectacle de la ville qui se déroule dedans et dehors.

Based on a theoretical framework that address the question of mediality, i.e. the sedimentation and circulation processes, and the production of urban culture, this article reflects on the case of the urban park of the Grande Bibliothèque, in downtown Montréal. The article is also inquiring into the current transformations of the public space and the mundane phenomenology it entails. It is suggested that the urban park is opening out to the city, in the midst of a contradictory discursive space. The article illustrates how the artistic strategies, the pressure of the urban network, the development and revitalization projects in the nearby area, the practices and the memories of the place, all of those dimensions are heading for the "reframing" of the park as meaningfull structure, and of the whole spectacle of the city spreading inside and outside.

\section{INDEX}

Keywords : urban park, culture, experience, mediality, public space

Mots-clés : jardin urbain, culture, expérience, médialité, espace public

\section{AUTEUR}

\section{LOUIS JACOB}

Louis Jacob est professeur de sociologie, département de sociologie, université du Québec à

Montréal.

jacob.louis[at]uqam[dot]ca 